\section{(1)}

CrossMark

\title{
Differential DNA methylation in bronchial biopsies between persistent asthma and asthma in remission
}

\author{
Cornelis J. Vermeulen (10 ${ }^{1,2}$, Cheng-Jian $\mathrm{Xu}^{2,3,4}$, Judith M. Vonk (10), \\ Nick H.T. ten Hacken ${ }^{1,2}$, Wim Timens ${ }^{2,6}$, Irene H. Heijink ${ }^{1,2,6}$, \\ Martijn C. Nawijn ${ }^{2,6}$, Jeunard Boekhoudt ${ }^{6}$, Antoon J. van Oosterhout ${ }^{7}$, \\ Karen Affleck ${ }^{7}$, Markus Weckmann $\mathbb{1 0}^{8}$, Gerard H. Koppelman (10) ${ }^{2,3}$ \\ and Maarten van den Berge $e^{1,2}$
}

\begin{abstract}
Affiliations: ${ }^{1}$ Dept of Pulmonary Diseases, University Medical Center Groningen, University of Groningen, Groningen, The Netherlands. ${ }^{2}$ University Medical Center Groningen, Groningen Research Institute for Asthma and COPD (GRIAC), University of Groningen, Groningen, The Netherlands. ${ }^{3}$ Dept of Pediatric Pulmonology and Pediatric Allergology, University of Groningen, University Medical Center Groningen, Beatrix Children's Hospital, Groningen, The Netherlands. ${ }^{4} \mathrm{CiiM} \&$ TWINCORE, Helmholtz-Centre for Infection Research (HZI) and the Hannover Medical School (MHH), Hannover, Germany. ${ }^{5}$ Dept of Epidemiology, University Medical Center Groningen, University of Groningen, Groningen, The Netherlands. ${ }^{6}$ Dept of Pathology and Medical Biology, University of Groningen, University Medical Center Groningen, Groningen, The Netherlands. ${ }^{7}$ Allergic Inflammation Discovery Performance Unit, GlaxoSmithKline, Stevenage, UK. ${ }^{8}$ Dept of Pediatric Pneumology and Allergology, University Medical Center of Schlesswig-Holstein, Airway Research Centre North, Member of the German Centre of Lung Research, Lübeck, Germany.
\end{abstract}

Correspondence: Cornelis J. Vermeulen, University of Groningen, University Medical Center Groningen, Dept of Pulmonary Diseases, NL-9700 RB Groningen, The Netherlands. E-mail: c.j.vermeulendumcg.nl

@ERSpublications

Former asthma patients have epigenetic modifications not present in current asthma which are associated with the activity of genes involved in the resolution of inflammation. Their epigenetic profile also shows them to be different from healthy controls. http://bit.ly/2BGmCPl

Cite this article as: Vermeulen CJ, Xu C-J, Vonk JM, et al. Differential DNA methylation in bronchial biopsies between persistent asthma and asthma in remission. Eur Respir J 2020; 55: 1901280 [https://doi. org/10.1183/13993003.01280-2019].

ABSTRACT Approximately $40 \%$ of asthmatics experience remission of asthma symptoms. A better understanding of biological pathways leading to asthma remission may provide insight into new therapeutic targets for asthma. As an important mechanism of gene regulation, investigation of DNA methylation provides a promising approach. Our objective was to identify differences in epigenome wide DNA methylation levels in bronchial biopsies between subjects with asthma remission and subjects with persistent asthma or healthy controls.

We analysed differential DNA methylation in bronchial biopsies from 26 subjects with persistent asthma, 39 remission subjects and 70 healthy controls, using the limma package. The comb-p tool was used to identify differentially methylated regions. DNA methylation of CpG-sites was associated to expression of nearby genes from the same biopsies to understand function.

Four CpG-sites and 42 regions were differentially methylated between persistent asthma and remission. DNA methylation at two sites was correlated in cis with gene expression at ACKR2 and DGKQ. Between remission subjects and healthy controls $1163 \mathrm{CpG}$-sites and 328 regions were differentially methylated. DNA methylation was associated with expression of a set of genes expressed in ciliated epithelium.

CpGs differentially methylated between remission and persistent asthma identify genetic loci associated with resolution of inflammation and airway responsiveness. Despite the absence of symptoms, remission subjects have a DNA methylation profile that is distinct from that of healthy controls, partly due to changes in cellular composition, with a higher gene expression signal related to ciliated epithelium in remission versus healthy controls. 


\section{Introduction}

Asthma is a chronic inflammatory disease of the airways, characterised by variable airflow obstruction associated with symptoms of wheezing, shortness of breath, chest tightness and coughing. Chronic inflammation in asthma is associated with remodelling of the airways, e.g. increased basal membrane thickness, airway epithelial shedding, increase of goblet cells, increased mucus production and increase of smooth muscle mass [1]. Interestingly, in some asthma patients, symptoms disappear over time, and the need for pulmonary medication ceases. The prevalence of asthma is highest between 10 and 25 years and gradually decreases at higher age, the latter indicating that asthma remission rates have become higher than incidence rates [2]. In adulthood, the average remission rate of asthma is $\sim 2 \%$ per year, with a higher chance to go into remission with an earlier onset of asthma, less severe airway obstruction and cessation of smoking $[3,4]$. A subset of patients with asthma in remission still have airway obstruction and/or show airway hyperresponsiveness (AHR) in provocation tests [5]. VONK et al. [6] introduced the terms "clinical remission" and "complete remission". Clinical remission is defined as the absence of asthma symptoms and no use of asthma medication. Complete remission of asthma is defined as the absence of asthma symptoms, no use of asthma medication, normal lung function and no AHR.

We still have minimal understanding of the cellular and molecular mechanisms that determine whether or not asthma persists or undergoes apparent spontaneous resolution. As for asthma development, genetic and environmental factors are likely to be involved in asthma remission as well [7]. The effect of both genetic and environmental variation on health outcome is often mediated by differences in gene transcription [8]. The methylation of cytosine at CpG-sites is an important epigenetic modification that regulates transcription by affecting several mechanisms [9]. Variation in DNA methylation at CpG-sites has been associated with asthma and other atopic diseases [10-15]. This warrants further investigation of this type of epigenetic variation.

In order to identify molecular mechanisms related to asthma remission in the airways, we investigated whether variation in DNA methylation in bronchial biopsies is associated with remission of asthma. Our main aim was to detect CpG-sites that are differentially methylated between well-characterised subjects in complete and clinical remission and subjects with persistent asthma or healthy controls. In addition, we investigated how differentially methylated CpG-sites associate with the expression levels of nearby genes in these bronchial biopsies, and related these to candidate molecular and cellular pathways. This furthers our ultimate aim to identify cellular mechanisms related to asthma remission in the airways.

\section{Methods}

Subjects

From previous studies $[16,17]$, biopsies were available from clinically well-characterised subjects with clinical remission (ClinR, $\mathrm{n}=33$ ), complete remission (ComR, $\mathrm{n}=15)$ and persistent asthma (PersA, $\mathrm{n}=90)$. Biopsies from healthy controls $(n=94)$ were available from another study. The study design and methods have been published previously $[16,17]$. The subjects with ClinR and ComR had a documented diagnosis of asthma, confirmed by AHR testing [6, 17]. Asthma was defined as documented reversibility and/or AHR to histamine (provocative concentration causing a $20 \%$ fall in forced expiratory volume in $1 \mathrm{~s}\left(\mathrm{FEV}_{1}\right)$ $\left.\left(\mathrm{PC}_{20}\right) \leqslant 32 \mathrm{mg} \cdot \mathrm{mL}^{-1}\right)$. Subjects were considered to be ClinR if they had not had an asthma attack or wheeze in the past 3 years, and did not use asthma medication ( $\beta$-agonists and inhaled corticosteroids (ICS)). Asthma patients were considered to be ComR if, in addition to the previous criteria, they did not have AHR to both histamine and AMP $\left(>32 \mathrm{mg} \cdot \mathrm{mL}^{-1}\right.$ in $30 \mathrm{~s}$ tidal breathing and $>320 \mathrm{mg} \cdot \mathrm{mL}^{-1}$ in $2 \mathrm{~min}$ tidal breathing, respectively) and had no signs of airflow obstruction ( $\mathrm{FEV}_{1} \%$ predicted $>80 \%$ pre-bronchodilator or $>90 \%$ post-bronchodilator). The subjects with PersA were divided on the basis of use of ICS, since corticosteroids are known to affect gene expression and may act as a confounder on DNA methylation as well [18]. The non-asthmatic healthy controls were recruited from the NORM study (Study to Obtain Normal Values of Inflammatory Variables from Healthy Subjects; clinical trials number NCT00848406) and were all current smokers and never-smokers aged $>18$ years. Subjects were considered healthy if they had no respiratory symptoms, no history of respiratory disease and normal pulmonary function, defined as a post-bronchodilator $\mathrm{FEV}_{1} /$ forced vital capacity ratio higher than the lower limit of normal, absence of AHR to methacholine $\left(\mathrm{PC}_{20}>16 \mathrm{mg} \cdot \mathrm{mL}^{-1}\right)$ and absence of $\mathrm{FEV}_{1}$ reversibility (increase in $\mathrm{FEV}_{1}$ after $400 \mu \mathrm{g}$ salbutamol $<10 \%$ pred). Healthy subjects were excluded if they had used inhaled or oral corticosteroids within the past 5 years, or during a total of 5 years of their lives. Biopsies from healthy subjects were collected between 2009 and 2012 and biopsies from all other subjects between 2005 and

This article has supplementary material available from erj.ersjournals.com

Received: 27 June 2019 | Accepted after revision: 18 Oct 2019 
2007. All subjects were included in the same centre (University Medical Center Groningen, Groningen, the Netherlands) and bronchoscopies and processing of samples were performed by the same team. For RNA and DNA extraction and further processing, an even distribution of case-control status, age, sex and smoking status among batches was carefully controlled during all steps in the experimental design. Details on the DNA/RNA extraction, sample preparation and quality control are provided in the supplementary methods. The study protocol was approved by the local medical ethics committee. All subjects gave their written informed consent.

\section{DNA methylation}

DNA methylation levels were measured in bronchial biopsies using the Infinium HumanMethylation450 BeadChip array (450 k array) (Illumina, San Diego, CA, USA). Raw intensity data were processed using the minfi package [19]. Samples and probes failing quality control were removed and raw $\beta$ values normalised using the dasen method as implemented in the wateRmelon package [20]. DNA methylation levels at each CpG-site were expressed as $\beta$-values, ranging from zero (no methylation) to one (complete methylation). A detailed description can be found in the supplementary methods. An overview of the sample dropout during quality control is shown in supplementary figure S1a.

\section{RNA sequencing}

RNA samples from airway wall biopsies were processed using the TruSeq Stranded Total RNA Sample Preparation Kit (Illumina, San Diego, CA, USA). The cDNA fragment libraries were loaded unto an Illumina HiSeq2500 sequencer for paired-end sequencing $(2 \times 100 \mathrm{bp})$. Trimmed fastQ files where aligned to build b37 of the human reference genome using HISAT (version 0.1.5) and gene level quantification was performed by HTSeq (version 0.6.1p1) using Ensembl version 75 as gene annotation database [21]. A detailed description can be found in the supplementary methods. An overview of the sample dropout during quality control is shown in supplementary figure S1b.

\section{Differential methylation analysis}

Differential methylation between subject groups was assessed for each probe using robust linear modelling in the limma package $[22,23]$. To remove heteroscedasticity, $\beta$-values were logit-transformed to M-values [24]. M-values were used as the dependent variable in all analyses. Age, sex and current smoking status were corrected for. In addition, the principal components explaining $95 \%$ of the variation in the control probes were used as covariates to correct for technical variation [25]. In addition, we analysed a model that included ex-smoker status in addition to current smoking, and found consistent results with the majority of differentially methylated regions (DMRs) remaining below the significance threshold. In remission versus asthma, of the 46 CpGs plus DMRs, 42 (91\%) remained significant. In remission versus healthy controls from the 1491 CpGs plus DMRs, 1223 (82\%) remained significant, but as this did not appreciably affect the results, these data are not presented. We analysed five subject categories: persistent asthma using ICS (PersA_ICS), persistent asthma not using ICS (PersA_no_ICS), ClinR, ComR and healthy controls. We pooled ClinR and ComR to increase sample size. We specified two contrasts: (ComR +ClinR) versus PersA_no_ICS and (ComR+ClinR) versus healthy controls. We refer to those contrasts as "remission versus asthma" and "remission versus healthy", respectively. To avoid the confounding of ICS use, we focused on the group of asthmatics without ICS. We applied correction for multiple testing by controlling the false discovery rate at $5 \%$ using the Benjamini-Hochberg method [26]. The most significant hits of individual $\mathrm{CpG}$-sites were annotated to the nearest gene using information from the IlluminaHumanMethylation450kanno.ilmn12.hg19 annotation package, or when no annotation was provided we searched the University of California Santa Cruz genome browser (https://genome.ucsc.edu) [27].

\section{Differentially methylated regions}

Regions of correlated CpG-sites with differential methylation were identified using the comb-p v0.48 command line tool and python library [28]. We used the pipeline with seed setting $\mathrm{p}=0.05$ and distance $750 \mathrm{bp}$. The region filter settings were $\mathrm{n}=2$ and a Sidak-corrected $\mathrm{p}=0.05$.

\section{Association between DNA methylation and gene expression (eQTM mapping)}

We used the MatrixEQTL package to determine whether DNA methylation levels at CpG-sites were associated with gene expression levels of nearby genes within $1 \mathrm{Mb}$ of the CpG-site [29]. Raw read counts from RNA sequencing were normalised for library size using the weighted trimmed mean of M-values method as implemented in the edgeR package [30]. The normalised counts were transformed to $\log 2$ (counts per million). We used methylation $\mathrm{M}$-values as the explanatory variable and age, sex and current smoking status were set as covariables. 


\section{Cell type composition}

Surrogate variable analysis provides estimates of latent variables, which is recommended as substitute for cell type deconvolution [31]. A detailed description can be found in the supplementary methods. We performed this analysis for all false discovery rate-significant differentially methylated CpGs from remission versus asthma and the 10 most differentially methylated CpGs from remission versus healthy.

We also took a targeted approach to the analysis of cell type composition, by extending the method proposed by $\mathrm{XU}$ et al. [13]. For the same set of CpG-sites as used in the surrogate variable analysis, we performed genome-wide mapping of DNA methylation to gene expression. For each CpG-site, the resulting set of test statistics was used as input for mean-rank gene set enrichment analysis as implemented in limma [32]. We tested enrichment of 20 gene sets expressed by the cell type clusters taken from single cell sequencing results of airway wall biopsies as reported in VIEIRA BRAGA et al. [33].

\section{Results}

\section{Patient characteristics}

We obtained DNA methylation data for 179 samples, after excluding 53 samples that were lost during processing or excluded during the quality control procedure (supplementary figure S1). Of the included samples, 12 subjects had ComR, 27 subjects ClinR, 70 subjects PersA and 70 subjects were healthy controls. Of the PersA subjects, 26 were not using ICS. The patient characteristics of the five subject groups are presented in table 1 .

\section{Differential methylation in remission versus persistent asthma}

Methylation levels at four individual CpG-sites and 42 regions were significantly different in remission versus asthma (tables 2 and 3). The most significant site was cg08364654, which showed 6\% lower methylation in remission subjects than in asthmatics (figure 1a). This CpG is also part of the most significant DMR on chromosome 3 (table 3). All CpGs in the DMR are located in the KRBOX1 gene, but we did not find these CpGs to be associated with KRBOX1 expression. Instead, a higher methylation was associated (most significantly at $\operatorname{cg} 22714811$ at $\mathrm{p}=7.9 \cdot 10^{-4}$ ) with increased expression of the atypical chemokine receptor 2 (ACKR2) gene (figures 2a and 3). The second CpG-site (cg23805470) also had 2\% lower methylation in subjects in remission compared to persistent asthmatics. Methylation of cg23805470 was not significantly associated with expression of the gene it resides in (tenascin $X B, T N X B$ ) or any other nearby gene. The third site cg13525448 lies in an exon of ladybird homeobox 1 (LBX1), but was not

\section{TABLE 1 Patient characteristics}

PersA_ICS PersA_no_ICS ClinR ComR Healthy controls $H_{0}$ : no differences between
groups. test-statistic; df; p-value

\begin{tabular}{|c|c|c|c|c|c|c|}
\hline Subjects $\mathrm{n}$ & 44 & 26 & 27 & 12 & 70 & $X^{2}=55.2 ; d f=4 ; p \ll 0.01$ \\
\hline $\mathrm{PC}_{20} \mathrm{AMP} \mathrm{mg} \cdot \mathrm{mL}^{-1}$ & 64.9 & 47.4 & 700.2 & 639.0 & 2674.9 & $F_{4-174}=10.9 ; P(e m p)=0.001$ \\
\hline & $(0.02-4871.5)$ & $(0.02-3811.4)$ & $(0.02-5090.7)$ & $(414.8-5635.8)$ & $(40-5612.4)$ & \\
\hline $\mathrm{FEV}_{1} \%$ pred & $83.1 \pm 3.02$ & $83.3 \pm 2.39$ & $90.8 \pm 2.70$ & $101.8 \pm 4.05$ & $101.0 \pm 1.34$ & $F_{4-174}=14.0-p \ll 0.001$ \\
\hline$\beta$-agonist use & 40 (91) & $14(54)$ & $0(0)$ & $0(0)$ & $0(0)$ & Chi-squared $=131.1 ; d f=4 ; p \ll 0.01$ \\
\hline Male/female & $20 / 24$ & $15 / 11$ & $12 / 15$ & $6 / 6$ & $40 / 30$ & Chi-squared $=2.47-d f=4-p=0.650$ \\
\hline Age years & $48.8 \pm 1.85$ & $46.3 \pm 2.44$ & $47.5 \pm 2.33$ & $45.3 \pm 4.78$ & $39.5 \pm 2.03$ & $F_{4-174}=3.37 ; p=0.011$ \\
\hline Atopy (skin-prick) & NA & NA & NA & NA & $25(36)$ & NA \\
\hline Atopy (phadiatop) & $31(70)$ & 19 (73) & $15(56)$ & $8(67)$ & NA & Chi-squared $=3.95-d f=3-p=0.267$ \\
\hline Smoking status & & & & & & Chi-squared=43.8; $d f=8 ; p \ll 0.001$ \\
\hline Current-smoker & 6 & 10 & 4 & 4 & 33 & \\
\hline Ex-smoker & 21 & 3 & 11 & 1 & 3 & \\
\hline Never-smoker & 17 & 13 & 12 & 7 & 34 & \\
\hline Blood eosinophils $\times 10^{9} \cdot \mathrm{L}^{-1}$ & $\begin{array}{c}0.195 \\
(0.05-0.78)\end{array}$ & $\begin{array}{c}0.260 \\
(0.00-0.90)\end{array}$ & $\begin{array}{c}0.170 \\
(0.00-0.62)\end{array}$ & $\begin{array}{c}0.115 \\
(0.00-0.28)\end{array}$ & $\begin{array}{c}0.155 \\
(0.02-0.43)\end{array}$ & $F_{4-174}=3.373 ; p=0.011$ \\
\hline
\end{tabular}

Data are presented as $n$, median (range) or mean \pm SEM, unless otherwise stated. PersA: persistent asthma; ICS: inhaled corticosteroids; ClinR: clinical remission; ComR: complete remission; df: degrees of freedom; $F E V_{1}$ : forced expiratory volume in $1 \mathrm{~s}$; $\mathrm{PC}_{20}$ : provocative concentration causing a $20 \%$ fall in $\mathrm{FEV}_{1}$; FVC: forced vital capacity. Frequency data were analysed using Chi-squared tests of independence. Differences in continuous variables were tested with one-way ANOVA. Variables were transformed, if necessary, to satisfy the assumption of normality. For $\mathrm{FEV}_{1}$ and $\mathrm{FEV}_{1} / \mathrm{FVC}$ ratio ANOVA was performed on z-values (GLI2012 DataConversion software). When $\mathrm{PC}_{20} \mathrm{AMP}$ was not reached at the highest dose, values were obtained by extrapolating from an exponential decay dose response curve. No appropriate transformation for $\mathrm{PC}_{20} \mathrm{AMP}$ was found, and these data were analysed by one-way ANOVA based on a permutated distribution of $\mathrm{F}$ with 1000 permutations [41]. 


\begin{tabular}{|c|c|c|c|c|c|c|c|c|c|}
\hline & Genomic position & $\begin{array}{c}\text { UCSC } \\
\text { RefGene } \\
\text { name }\end{array}$ & $\begin{array}{l}\text { UCSC } \\
\text { RefGene } \\
\text { group }\end{array}$ & Correlated expression & $\log \mathrm{FC}$ & $\begin{array}{l}\text { Moderated } \\
\text { t-value }\end{array}$ & p-value & $\begin{array}{l}\text { Adjusted } \\
\text { p-value }\end{array}$ & $\begin{array}{c}\text { SVA } \\
\text { corrected } \\
\text { p-value }\end{array}$ \\
\hline \multicolumn{10}{|c|}{$\begin{array}{l}\text { Remission } \\
\quad \text { versus asthma }\end{array}$} \\
\hline cg08364654 & chr3:42,978,180 & & & & 0.83 & 6.23 & $3.5 \times 10^{-9}$ & 0.0015 & $6.5 \times 10^{-8}$ \\
\hline cg23805470 & chr6:32,056,820 & TNXB & Body & & 0.60 & 6.07 & $7.9 \times 10^{-9}$ & 0.0017 & $1.1 \times 10^{-7}$ \\
\hline cg13525448 & chr10:102,986,601 & & & & -0.48 & -5.26 & $4.4 \times 10^{-7}$ & 0.0466 & $3.2 \times 10^{-7}$ \\
\hline cg00741675 & chr4:967,327 & DGKQ & $\begin{array}{l}5^{\prime} \text { UTR; } 1 \text { st } \\
\text { exon }\end{array}$ & & 2.96 & 5.28 & $3.8 \times 10^{-7}$ & 0.0466 & $4.5 \times 10^{-4}$ \\
\hline \multicolumn{10}{|c|}{$\begin{array}{l}\text { Remission } \\
\quad \text { versus healthy }\end{array}$} \\
\hline cg04886217 & chr16:31,821,538 & & & & 0.63 & 6.56 & $6.1 \times 10^{-10}$ & 0.0003 & $2.8 \times 10^{-8}$ \\
\hline cg06947286 & chr5:131,596,602 & PDLIM4 & Body & PDLIM4; P4HA2; UQCRQ; HSPA4; KIF3A; ACSL6; ZCCHC10 & -0.60 & -6.20 & $4.2 \times 10^{-9}$ & 0.0006 & $6.8 \times 10^{-5}$ \\
\hline cg01716603 & 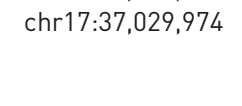 & LASP1 & Body & $\begin{array}{l}\text { SRCIN1; PLXDC1; ARHGAP23; HNF1B; LINC00672; PIP4K2B; } \\
\text { MLLT6; NEUROD2; STARD3; PCGF2; ENSG00000214546; } \\
\text { CACNB1 }\end{array}$ & -0.83 & -6.19 & $4.3 \times 10^{-9}$ & 0.0006 & $1.7 \times 10^{-5}$ \\
\hline $\operatorname{cg} 23932332$ & $\operatorname{chr} 1: 221,911,278$ & DUSP10 & $\begin{array}{l}\text { TSS1500; } \\
\text { body; 5'UTR }\end{array}$ & ENSG00000225265; FAM177B; MARC2; DUSP10 & -0.87 & -6.01 & $1.1 \times 10^{-8}$ & 0.0011 & 0.0062 \\
\hline $\operatorname{cg} 18763536$ & 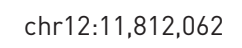 & ETV6 & Body & MANSC1; CREBL2; ETV6 & -0.44 & -5.97 & $1.4 \times 10^{-8}$ & 0.0011 & $1.8 \times 10^{-5}$ \\
\hline cg23916878 & chr6:44,011,187 & & & $\begin{array}{c}\text { DLK2; LRRC73; RSPH9; DNPH1; TCTE1; SPATS1; PTK7; } \\
\text { ENSG00000272442 }\end{array}$ & -0.68 & -5.94 & $1.6 \times 10^{-8}$ & 0.0011 & $2.9 \times 10^{-5}$ \\
\hline $\operatorname{cg} 20001791$ & chr6:16,239,799 & GMPR & Body & GMPR; STMND1 & -0.66 & -5.88 & $2.1 \times 10^{-8}$ & 0.0013 & $4.2 \times 10^{-7}$ \\
\hline cg08307963 & chr1:147,245,485 & GJA5 & TSS200 & BCL9 & 0.54 & 5.78 & $3.4 \times 10^{-8}$ & 0.0017 & $2.0 \times 10^{-7}$ \\
\hline cg10986462 & chr10:135,340,539 & CYP2E1 & TSS1500 & FUOM & -1.12 & -5.77 & $3.6 \times 10^{-8}$ & 0.0017 & $1.2 \times 10^{-5}$ \\
\hline cg07224931 & chr12:121,130,567 & MLEC & Body & DYNLL1-AS1; CIT; MORN3; UNC119B; PXN; DYNLL1 & -0.53 & -5.73 & $4.5 \times 10^{-8}$ & 0.0017 & $7.1 \times 10^{-5}$ \\
\hline
\end{tabular}

The table shows the probe ID, with its genomic position (hg19), the name of any nearby genes and its position relative to that gene, proximate genes with associated expression, the log 2 fold change (logFC) of the $\beta$-values, the moderated t-value and its associated probability of the null hypothesis of no difference between the groups, as well as the adjusted p-value by Benjamini-Hochberg correction controlling the false discovery rate at $5 \%$. The rightmost column lists the p-values of the analysis where surrogate variables were added as covariates (see text). Positive logFC indicates higher methylation in asthmatics than remission subjects and higher methylation in remission subjects than healthy controls. UCSC: University of California Santa Clara; SVA: surrogate variable analysis; UTR: untranslated region. 


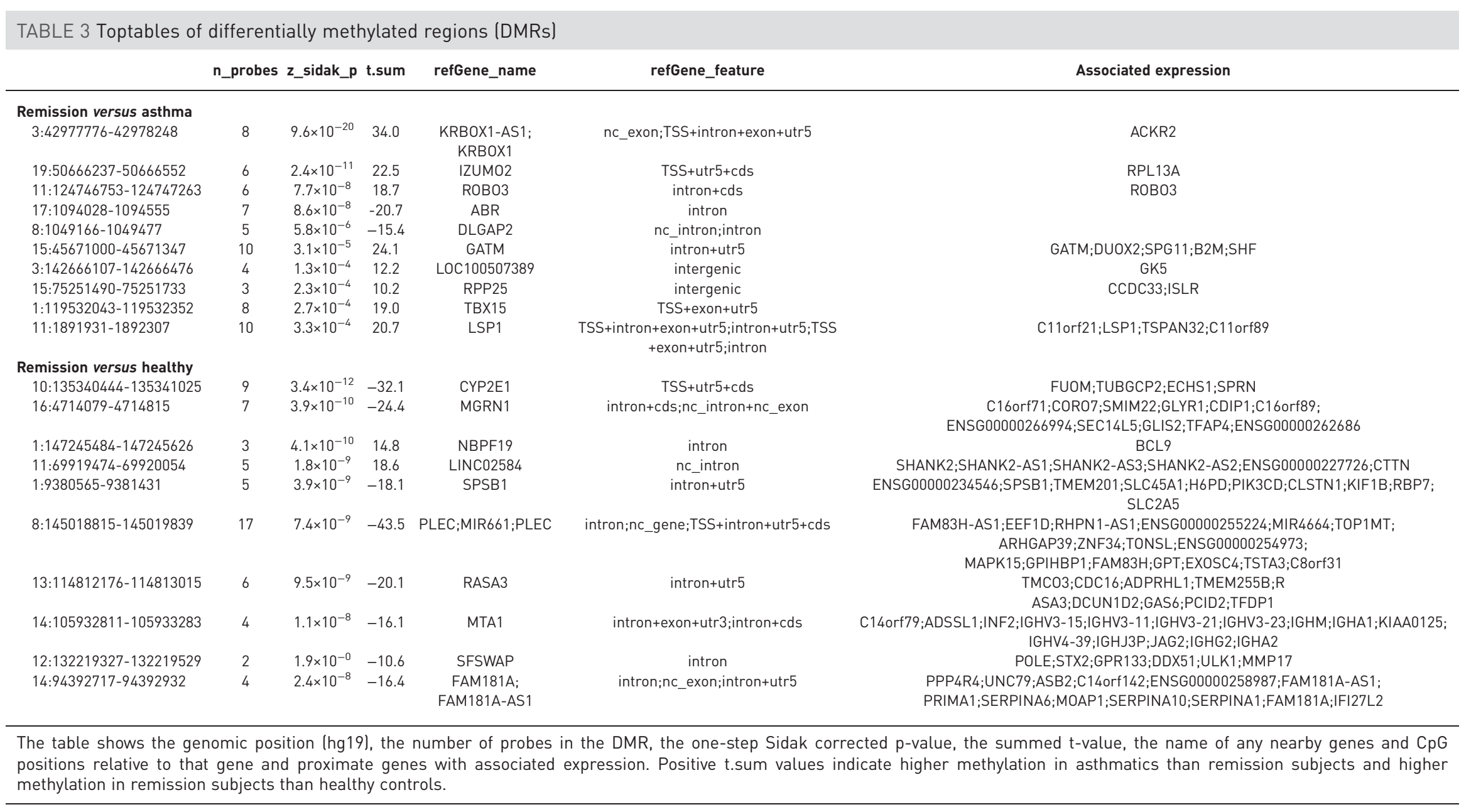


a)

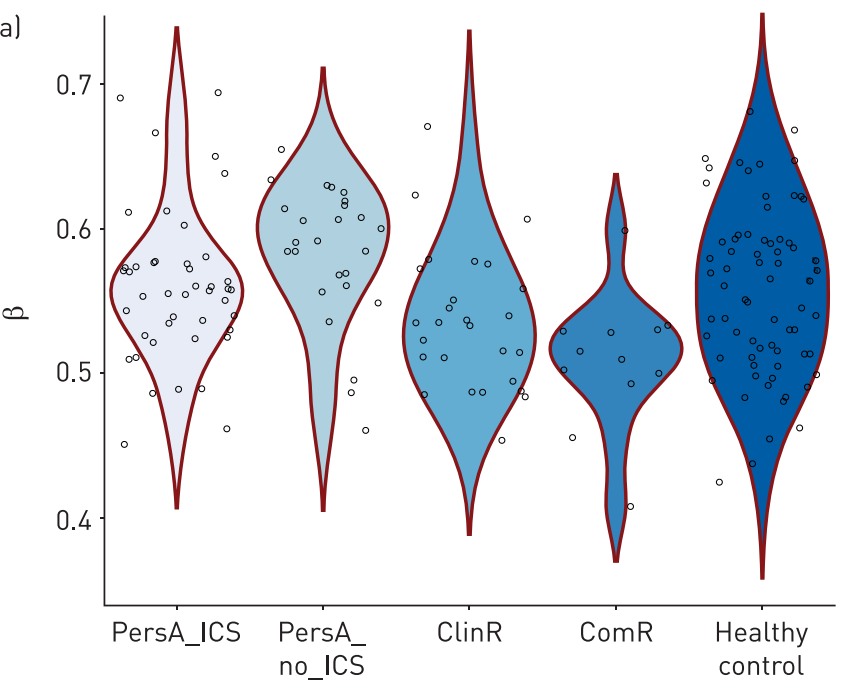

c)

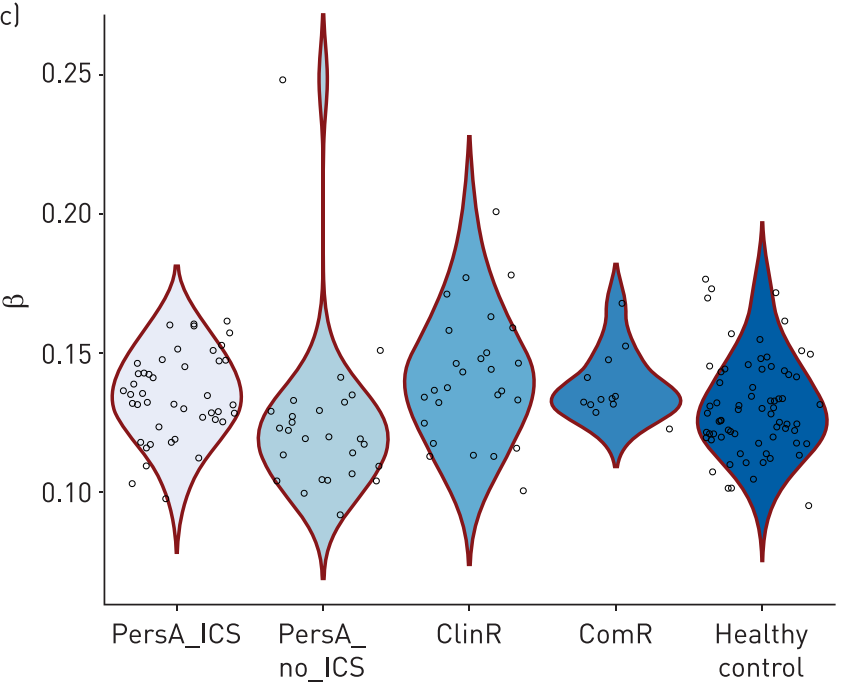

b)

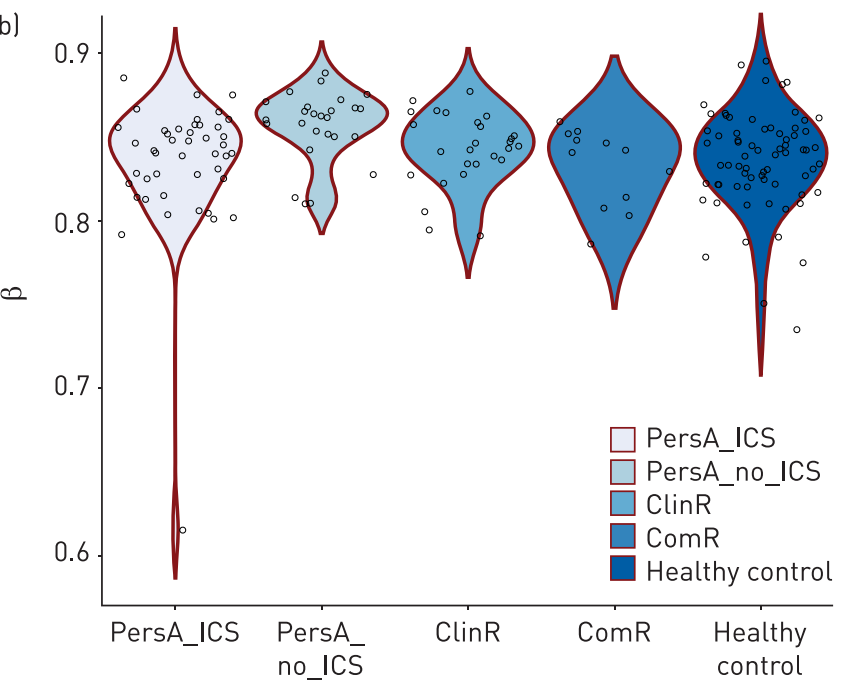

d)

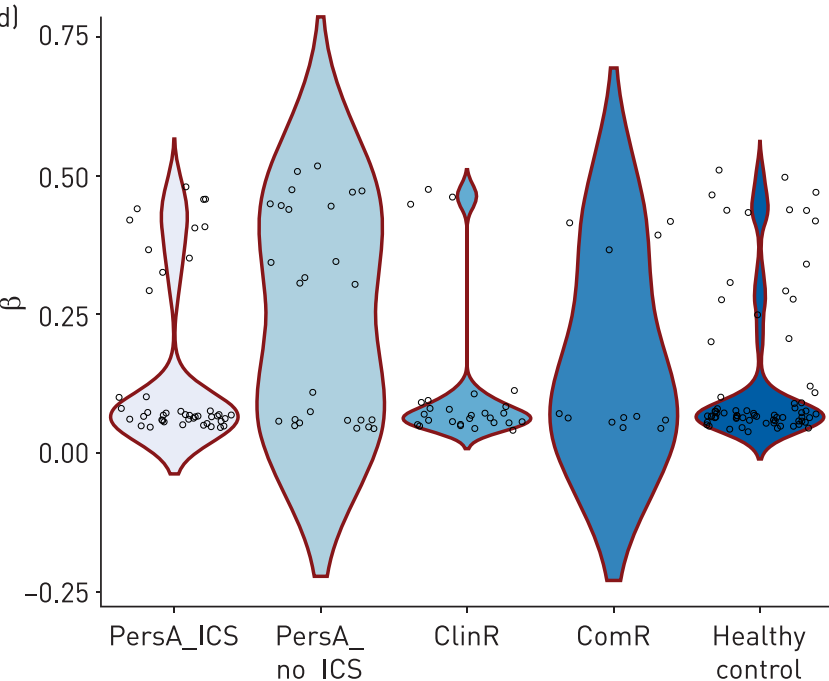

FIGURE 1 Violin plots of highly differentially methylated CpG-sites in the "asthma versus remission" contrast. a) cg08364654 in the KRBOX1 gene; b) cg23805470 in the TNXB gene; c) cg13525448; and d) cg00741675 in the DGKQ gene. PersA ICS: persistent asthma with inhaled corticosteroid (ICS) use; PersA_no_ICS: persistent asthma without ICS use; ClinR: clinical remission; ComR: complete remission.

associated with gene expression in cis. It had $2 \%$ higher methylation in remission than in persistent asthma (figure 1c). The fourth CpG-site (cg00741675) had 11\% lower methylation in remission and is located in the $5^{\prime}$ untranslated region of the diacylglycerol kinase, $\theta 110 \mathrm{kDa}(D G K Q)$ gene. It did not associate with gene expression at the $\mathrm{p}=0.001$ cut-off, but is positively associated with gene expression of $D G K Q$ at $\mathrm{p}<0.004$ (figure $2 \mathrm{~b}$ ). The second significant DMR is located at chromosome 19 and associated with expression of RPL13A. The encoded product is a component of the interferon- $\gamma$-activated inhibitor of translation complex, which plays a role in the repression of inflammatory genes and contributes to the resolution of chronic inflammation [34]. Among the four significant individual CpG-sites from the contrast remission versus asthma (table 2), all remained significantly differentially methylated (at $\mathrm{p}<0.001$ ) after correction for the first five surrogate variables, indicating that the differences in methylation were not driven by cell type composition (table 2 and supplementary figure S2a). Significance of cg00741675 dropped sharply, but for all four CpGs log fold changes remained similar after adjustment.

\section{Differential methylation in remission versus healthy controls}

We found 1163 individual CpG-sites and 328 regions to be differentially methylated between remission subjects and healthy controls, with one-third of the individual sites and $20 \%$ of the DMRs being higher methylated in remission (tables 2 and 3 show the top 10; complete list in supplementary tables S1B and S2B). Of the 1163 significant CpG-sites, 167 were located in a DMR. Correction for surrogate variables resulted in similar log fold changes, but did appreciably affect the significance of the 10 most significant 

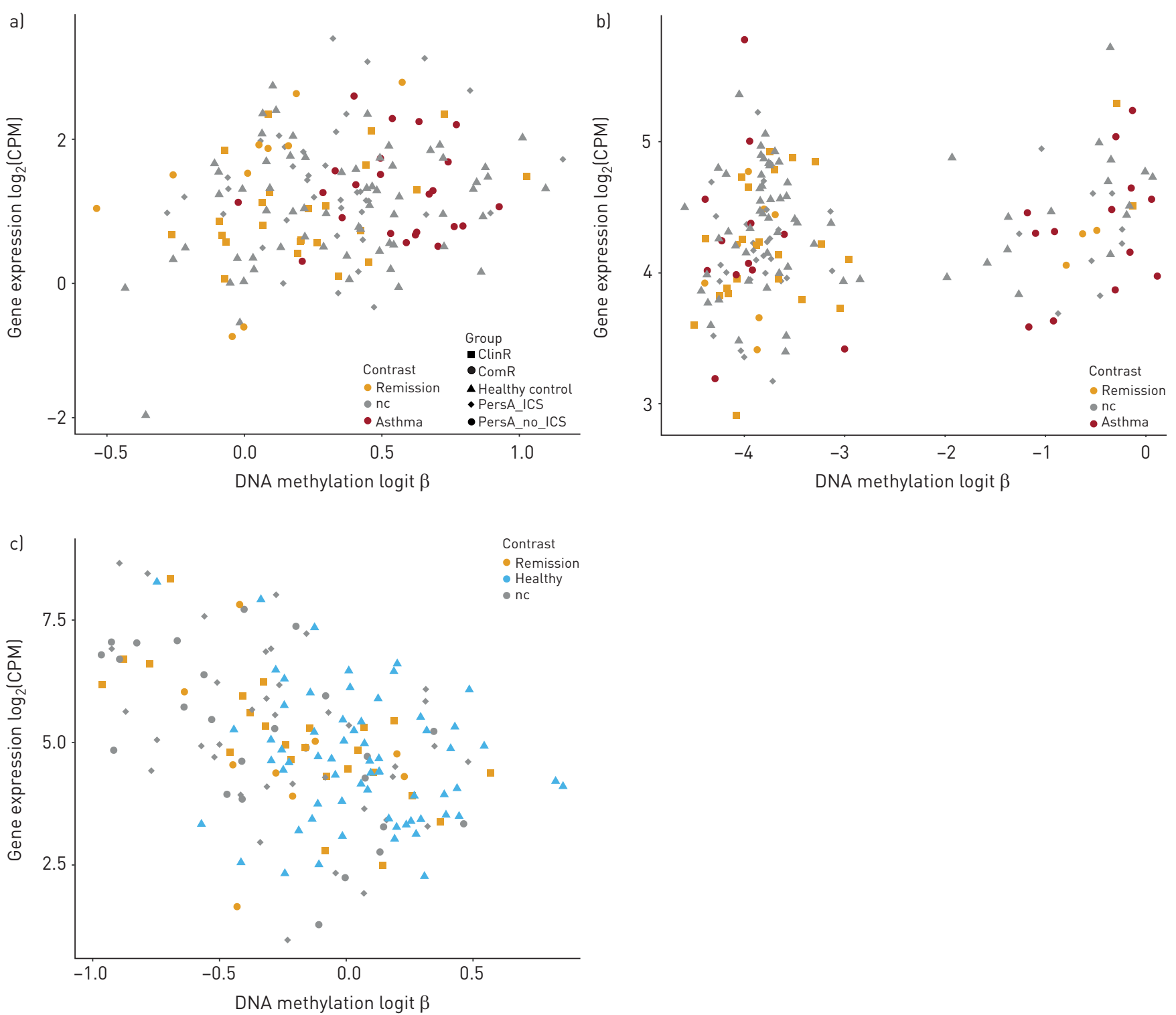

FIGURE 2 Correlation of DNA methylation at differentially methylated CpG-sites with gene expression of nearby genes. a) cg08364654 with ACKR2 expression; b) cg00741675 with DGKQ expression; c) cg18091275 with NOS2 expression. The groups used in the contrasts (either "remission versus asthma" or "remission versus healthy") are marked with non-grey colours. nc: no contrast; PersA_ICS: persistent asthma with inhaled corticosteroid (ICS) use; PersA_no_ICS: persistent asthma without ICS use; ClinR: clinical remission; ComR: complete remission.

CpG-sites, suggesting an impact of cell type composition in the contrast remission versus healthy (supplementary figure S2).

To further investigate effects of cell type composition we identified cell type specific gene expression profiles by associating methylation levels of the 10 most significant CpG-sites to genome-wide gene expression levels. Then we compared associated genes to gene sets characteristic for 20 major clusters of cells found in bronchial biopsies based on single cell sequencing data [33]. All 10 tested CpG-sites from the contrast remission versus healthy showed strong enrichment for association with genes belonging to multiple sets of cell types (supplementary table S3). The highest enriched groups throughout were ciliated epithelial cells and basal cells. However, several CpGs were most significantly enriched for other groups: smooth muscle cells (cg06947286 and cg10986462), fibroblasts (cg23916878), neutrophils (cg18763536) and T-cells (cg08307963) (figure 4a). Nine out of 10 tested CpG-sites from the contrast remission versus healthy controls showed strong enrichment $(\mathrm{p} \ll 0.001)$ for highly ranking genes from ciliated epithelium (figure 4). We tested whether the enrichment was due to the underlying correlation structure of our dataset, but this did not fully account for our findings. For comparison, only $49 \%$ of a randomly selected sample of CpG-sites has enrichment for this gene set at this significance level. In addition, five out of 10 


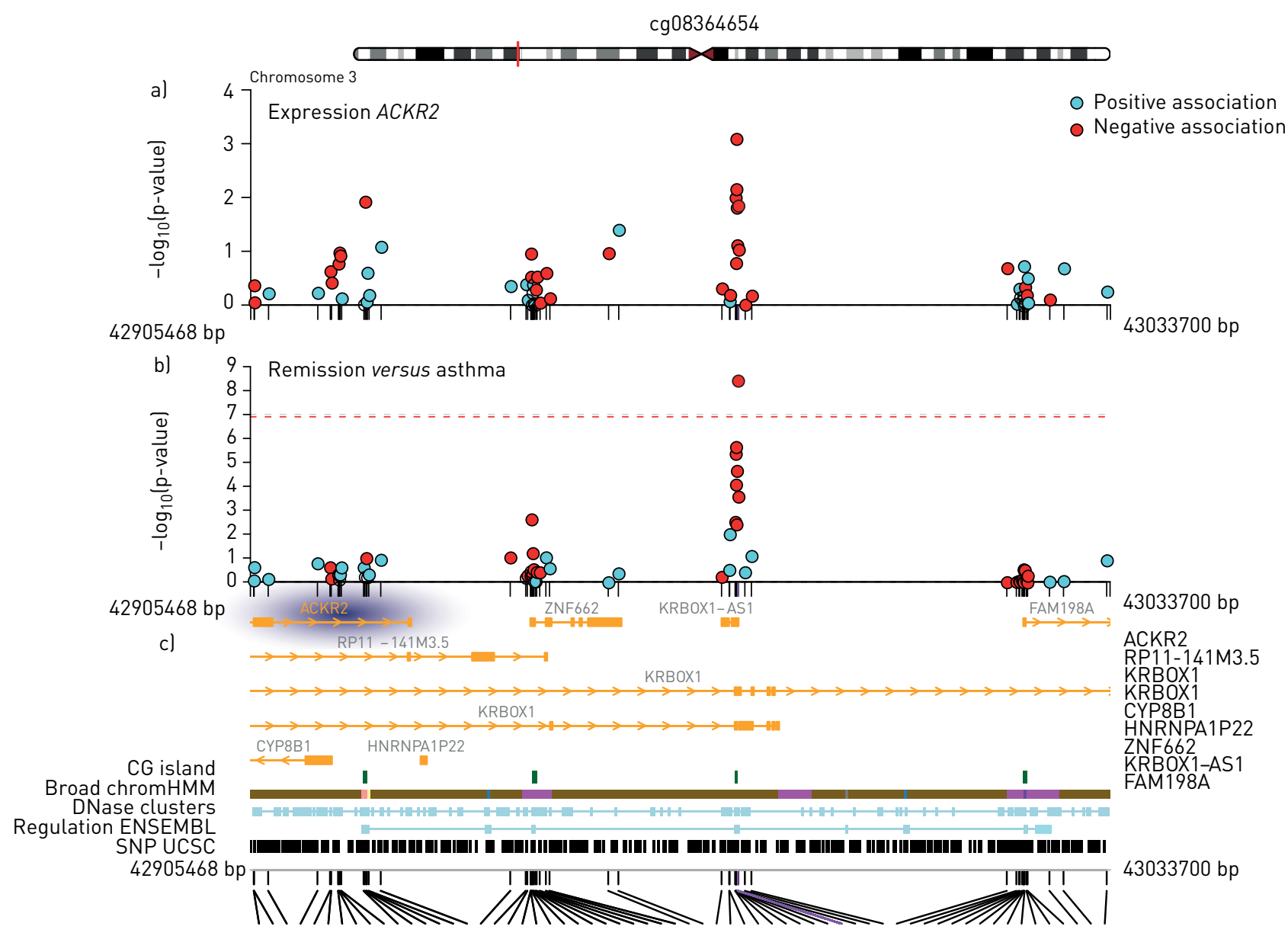

d)

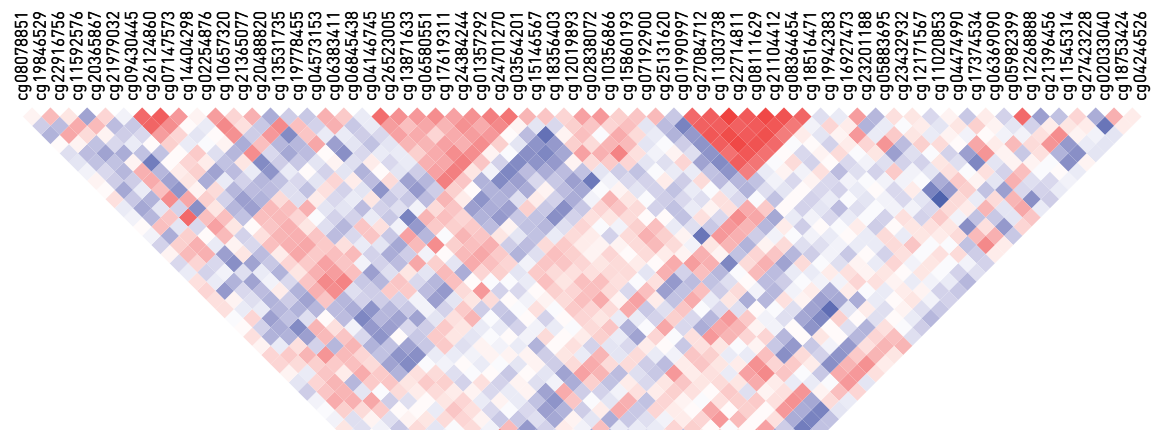

Physical distance: $128.2 \mathrm{~kb}$

Correlation matrix map type: Spearman

$1 \quad 0.6 \quad 0.2 \quad-0.2 \quad-0.6 \quad-1$

- $\mathrm{CpG}$
cg08364654

FIGURE 3 CoMET plot of the genomic region of cg08364654. a) The strength of correlation with expression of the ACKR2 gene for all the CpG-sites in the interval; b) the strength of association in the remission versus asthma contrast; c) default coMET annotation tracks: ENSEMBL genes, CpG islands, chromatin-state (Broad institute), DNAse-sensitive regions and SNPs (see coMET documentation for detailed description: https:// bioconductor.org/packages/release/bioc/html/coMET.html); d) correlation matrix of DNA methylation among all plotted CpG-sites.

tested CpG-sites belong to the top $1 \%$ enrichment as judged by comparison to p-values in the randomly selected sample. Figure $4 \mathrm{~b}$ shows the ranking of correlated genes of a representative $\mathrm{CpG}$-site from this set (cg07224931). Nearly all genes in the set related to ciliated epithelium have higher gene expression in remission versus healthy controls (99\%). 

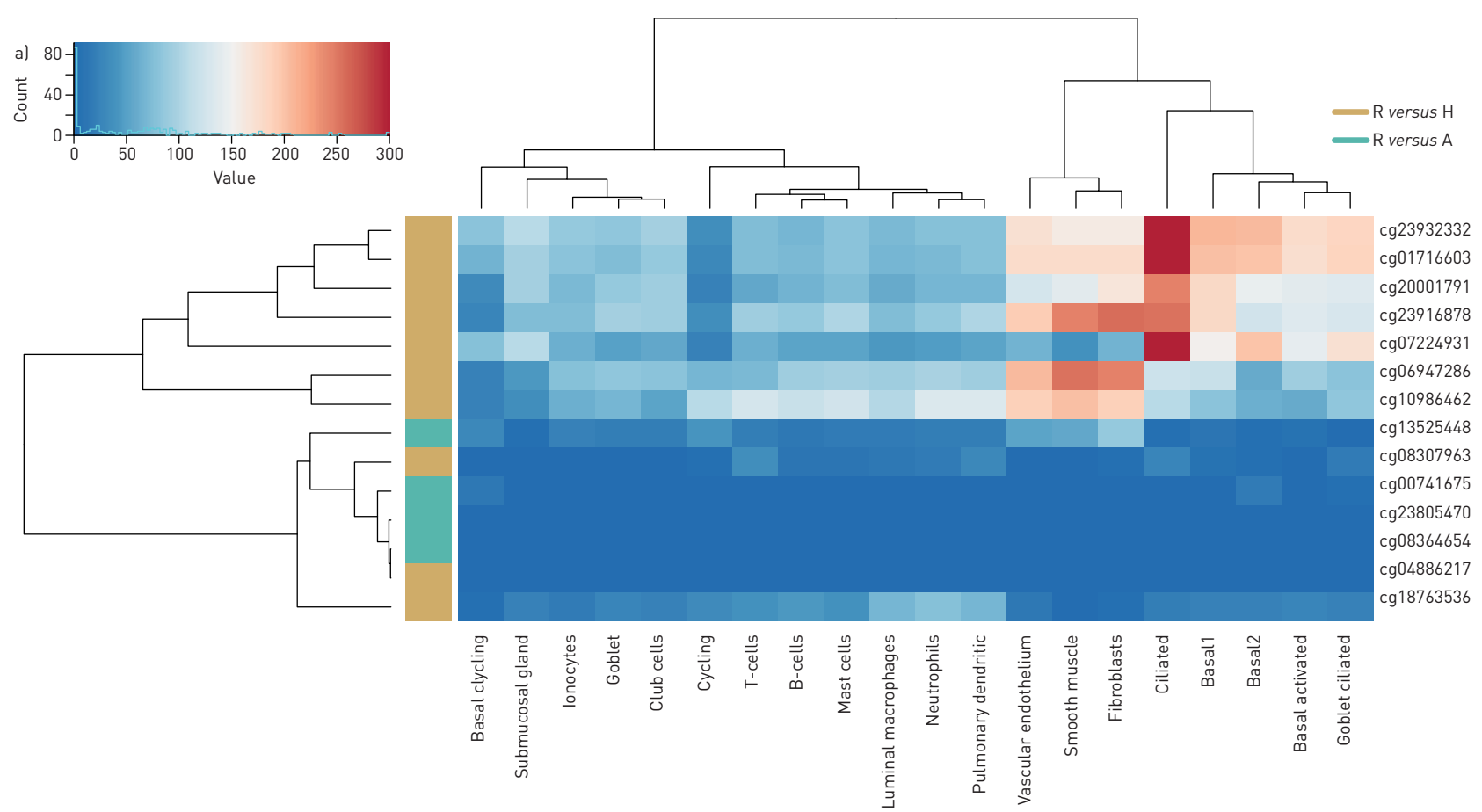

b)

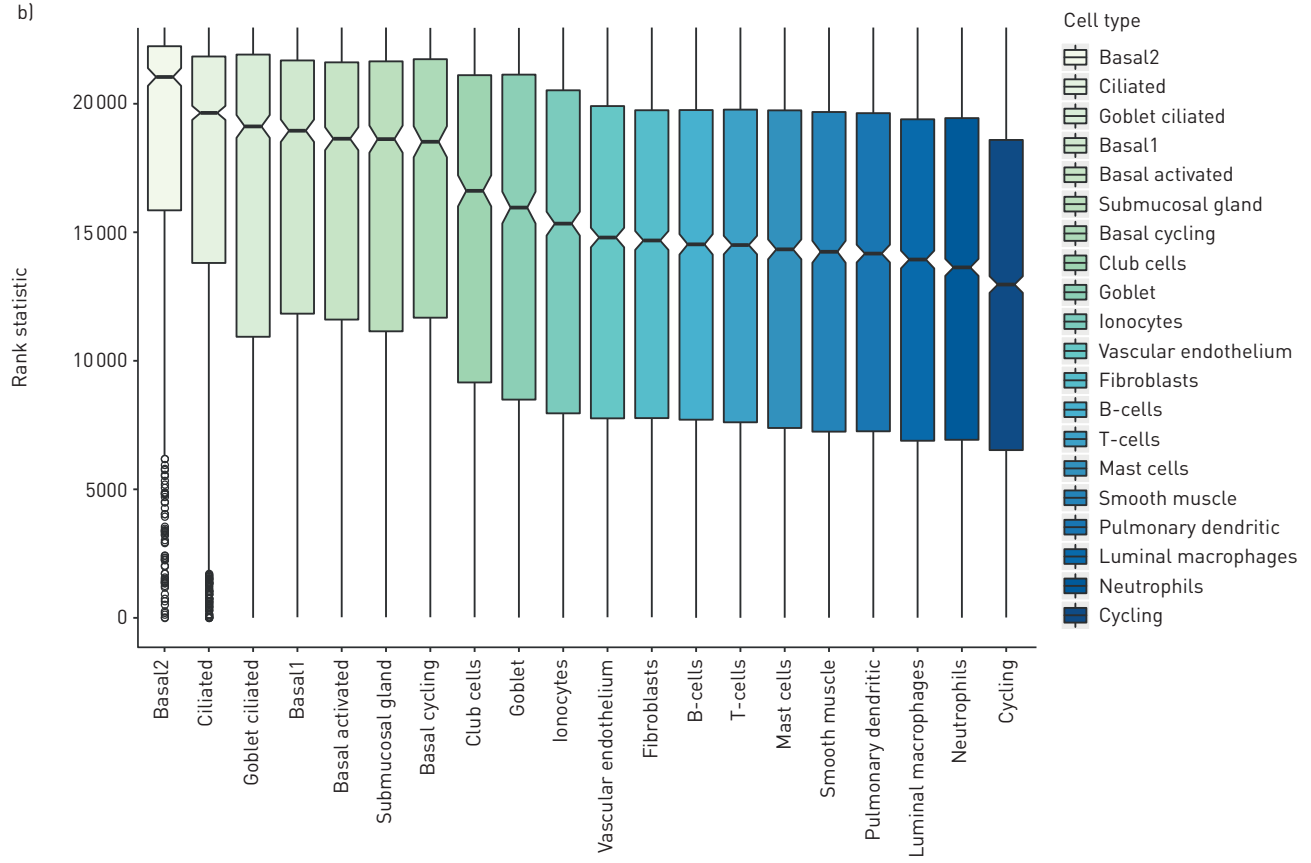

FIGURE 4 Effect of cell type composition on average methylation levels. Effect of cell type composition was gauged by genome wide correlations with gene expression. a) Heatmap and hierarchical clustering of $-\log _{10}$ (p-values) of mean-rank gene set tests on the test statistics of DNA methylation-gene expression associations. The $\mathrm{CpG}$-sites are the four most significant sites from the contrast remission versus asthma (R versus A) and the 10 most significant sites from the contrast remission versus healthy (R versus $H$ ). The gene sets distinguish the four main clusters of epithelial cell types in single cell sequencing. See text for details. b) Ranks of test statistic for a selected CpG-site (cg07224931) demonstrates the enrichment of highly ranking genes of the ciliated epithelium.

\section{Discussion}

We found four individual CpG-sites and 42 regions to be differentially methylated between subjects in remission and persistent asthmatics. Of the individual CpGs two associated with gene expression of $A C K R 2$ and $D G K Q$, respectively. These four CpGs were not strongly related to cell type composition of the airway wall biopsy. There were $1163 \mathrm{CpG}$-sites and 328 regions differentially methylated between 
remission subjects and healthy controls. Many of the highly significant CpG-sites that distinguish remission subjects from healthy controls were associated with increases in the expression of genes associated with ciliated epithelial cells, consistent with cell type composition differences in remission versus healthy controls. We found differences in absolute methylation between groups between $2 \%$ and $11 \%$. It should be noted that even modest differences can be relevant when they reflect changes within a specific cell type.

The most significant CpG site, as well as DMR, with lower methylation in the contrast remission versus asthma was associated with lower expression of ACKR2 in subjects with remission. This gene contributes to resolution of inflammation by post-inflammatory clearance of chemokines in a mouse model [35]. Allergen-challenged mice that are deficient for ACKR2 have more infiltrating cells in the airways, primarily dendritic cells and eosinophils. Interestingly, these ACKR2-deficient mice have less airway reactivity to methacholine [36]. We suggest that lower expression of ACKR2 may play a role in the remission of asthma, by reducing AHR. Of interest, the lower methylation and ACKR2 gene expression was paralleled by decreased airway reactivity in the $A C K R 2$-deficient mice, but not by reduced inflammation as would be expected. The CpG-site in TNXB could not be related to expression of this gene, but tenascin genes are known to be important in airway remodelling [1]. Multiple CpG-sites in TNXB were less methylated in primary airway epithelium cells after exposure to interleukin-13, a type 2 cytokine and key mediator of airway inflammation and remodelling in asthma [15]. TNXB resides in the human leukocyte antigen (HLA) class II region, which is important for immune function [37], but we were unable to link differential methylation at cg23805470 to expression of HLA genes. CpG-site cg00741675 in DGKQ has lower methylation in remission subjects when compared to persistent asthmatics. DGKQ encodes a member of the family of diacylglycerol kinases (DGKs) that attenuate levels of the second messenger diacylglycerol in cells by converting it to phosphatidic acid [38]. The precise mechanism by which it may be involved in the remission of asthma is not clear, but members of the DGK family are expressed in immune cells, and thus may be required for proper cellular signalling in inflammatory processes [39].

Subjects in remission have a methylation profile that is distinct from that of persistent asthmatics, which may reflect differential regulation of immune functions. However, their methylation profile does not resemble the healthy situation either. Individuals with complete asthma remission retain characteristics that differ from healthy subjects without a history of asthma, for example, persistent airway remodelling as shown by increased basal membrane thickness [17]. Analogous to this situation, we find methylation differences between remission and healthy controls that do not occur between remission and asthma. We found that 21 out of 42 DMRs and three out of four differentially methylated CpGs in remission versus asthma overlapped with DMRs found in asthma versus healthy controls. For remission versus healthy controls this concerned 126 out of 327 DMRs and 310 out of 1163 differentially methylated CpGs. This clearly shows that differential methylation in both the remission versus asthma and the remission versus healthy controls contrasts capture aspects of methylation differences between persistent asthmatics and healthy subjects, consistent with the intermediate position of remission subjects. A possible explanation is that subjects in asthma remission still display an epigenetic fingerprint of asthma. A similar lingering difference in methylation profiles was reported for ex-smokers [40]. Alternatively, this may reflect an inherent predisposition to developing asthma, which puts remission subjects at an increased risk for relapse.

A strength of our design is the use of bronchial biopsies, which enabled us to study epigenetic profiles in the relevant tissue. In addition, we included healthy subjects and well-characterised subjects in clinical and complete remission. All remission subjects had previous objective confirmation of their asthma diagnosis with detailed follow-up. Finally, we added functional information to differentially methylated CpG-sites by their association with the expression levels of nearby genes, using the same biopsy as used for determination of methylation levels. One limitation is the absence of an independent replication cohort in which bronchial biopsies are available for remission subjects and healthy controls. To the best of our knowledge, no comparable replication cohort with a similar design is available. Another issue is that differential methylation among subject groups was partly driven by differences in cell type composition, which hampers identification of specific asthma or remission related CpG-sites. By annotating the associated CpGs with gene expression, we show that subjects in remission differ from healthy controls in methylation levels at CpG-sites associated with multiple cell types, and that there is strong enrichment of $\mathrm{CpGs}$ in genes associated with ciliated epithelium. A possible interpretation is that changes in the cell type composition, in particular the proportion of ciliated epithelium in remission subjects explains part of the differential methylation between remission subjects and healthy controls. This is supported by the inflation of the p-values in this particular contrast. The prominent signal from epithelium may reflect the abundance of this cell type in biopsies, and the importance of other cell types in asthma and remission should not be neglected, but it emphasises that the airways of subjects in remission still have not come to resemble the healthy profile. 
Acknowledgements: We wish to thank Pieter van der Vlies, Bahram Sanjabi, Desiree Brandenburg-Weening and Harold de Bruin (University Medical Center Groningen, Groningen, the Netherlands) for their valuable work in the DNA extraction, sample preparation and hybridisation.

Author contributions: C.J. Vermeulen performed differential methylation analysis and drafted and finalised the manuscript, C-J. Xu and J.M. Vonk were involved in data management, data analysis and editing the manuscript, N.H.T. ten Hacken, W. Timens, I.H. Heijink, M.C. Nawijn, A. J. van Oosterhout, K. Affleck and G.H. Koppelman provided guidance and technical knowledge and edited the manuscript, J. Boekhoudt performed eQTM analysis, M. Weckmann contributed expression and methylation data, M. van den Berge, N.H.T. ten Hacken, W. Timens and J.M. Vonk were involved in the design of the study and M. van den Berge drafted parts of the manuscript. All authors approved the final manuscript.

Conflict of interest: C.J. Vermeulen reports grants from GSK, during the conduct of the study. C-J. Xu has nothing to disclose. J.M. Vonk has nothing to disclose. N.H.T. ten Hacken has nothing to disclose. W. Timens reports personal fees from Pfizer, GSK, Chiesi, Roche Diagnostics/Ventana, Biotest, Merck Sharp Dohme, Novartis, Lilly Oncology, Boehringer Ingelheim, AstraZeneca, Bristol-Myers-Squibb and AbbVie, and grants from the Dutch Asthma Fund, outside the submitted work. I.H. Heijink has nothing to disclose. M.C. Nawijn reports grants from GSK and the Lung Foundation of the Netherlands, outside the submitted work. J. Boekhoudt has nothing to disclose. A.J. van Oosterhout reports and holds GSK shares. K. Affleck is an employee of GSK and a member of the company share schemes. M. Weckmann has nothing to disclose. G.H. Koppelman reports grants from the Lung Foundation of the Netherlands, TEVA, GSK, Vertex and Ubbo Emmius Foundation, outside the submitted work; and has served on an international advisory board for GSK (money to institution). M. van den Berge reports grants paid to his university from AstraZeneca, TEVA, GSK and Chiesi, outside the submitted work.

Support statement: Data were generated as part of a Scientific Research Collaboration funded by GSK (COL100037293). The submitted work is co-financed by the Ministry of Economic Affairs and Climate Policy by means of the PPP. Funding information for this article has been deposited with the Crossref Funder Registry.

\section{References}

Holgate ST, Wenzel S, Postma DS, et al. Asthma. Nat Rev Dis Primers 2015; 1; 15025.

Upham JW, James AL. Remission of asthma: the next therapeutic frontier? Pharmacol Ther 2011; 130: 38-45.

Holm M, Omenaas E, Gíslason T, et al. Remission of asthma: a prospective longitudinal study from northern Europe (RHINE study). Eur Respir J 2007; 30: 62-65.

4 Carpaij OA, Nieuwenhuis MAE, Koppelman GH, et al. Childhood factors associated with complete and clinical asthma remission at 25 and 49 years. Eur Respir J 2017; 49: 1601974.

5 Boulet LP, Turcotte H, Brochu A. Persistence of airway obstruction and hyperresponsiveness in subjects with asthma remission. Chest 1994; 105: 1024-1031.

6 Vonk JM, Postma DS, Boezen HM, et al. Childhood factors associated with asthma remission after 30 year follow up. Thorax 2004; 59: 925-929.

7 Vonk JM, Nieuwenhuis MAE, Dijk FN, et al. Novel genes and insights in complete asthma remission: a genome-wide association study on clinical and complete asthma remission. Clin Exp Allergy 2018; 48: 1286-1296.

8 Edwards SL, Beesley J, French JD, et al. Beyond GWASs: illuminating the dark road from association to function. Am J Hum Genet 2013; 93: 779-797.

9 Reddington JP, Pennings S, Meehan RR. Non-canonical functions of the DNA methylome in gene regulation. Biochem J 2013; 451: 13-23.

10 DeVries A, Vercelli D. Early predictors of asthma and allergy in children: the role of epigenetics. Curr Opin Allergy Clin Immunol 2015; 15: 435-439.

11 Sabounchi S, Bollyky J, Nadeau K. Review of environmental impact on the epigenetic regulation of atopic diseases. Curr Allergy Asthma Rep 2015; 15: 33.

12 Miller RL, Peden DB. Environmental effects on immune responses in patients with atopy and asthma. J Allergy Clin Immunol 2014; 134: 1001-1008.

13 Xu C-J, Söderhäll C, Bustamante M, et al. DNA methylation in childhood asthma: an epigenome-wide meta-analysis. Lancet Respir Med 2018; 6: 379-388.

14 Nicodemus-Johnson J, Myers RA, Sakabe NJ, et al. DNA methylation in lung cells is associated with asthma endotypes and genetic risk. JCI Insight 2016; 1: e90151.

15 Nicodemus-Johnson J, Naughton KA, Sudi J, et al. Genome-wide methylation study identifies an IL-13 induced epigenetic signature in asthmatic airways. Am J Respir Crit Care Med 2016; 193: 376-385.

16 Broekema M, Volbeda F, Timens W, et al. Airway eosinophilia in remission and progression of asthma: accumulation with a fast decline of $\mathrm{FEV}_{1}$. Respir Med 2010; 104: 1254-1262.

17 Broekema M, Timens W, Vonk JM, et al. Persisting remodelling and less airway wall eosinophil activation in complete remission of asthma. Am J Respir Crit Care Med 2011; 183: 310-316.

18 van den Berge M, Steiling K, Timens W, et al. Airway gene expression in COPD is dynamic with inhaled corticosteroid treatment and reflects biological pathways associated with disease activity. Thorax 2014; 69: 14-23.

19 Aryee MJ, Jaffe AE, Corrada-Bravo H, et al. Genome analysis Minfi: a flexible and comprehensive Bioconductor package for the analysis of Infinium DNA methylation microarrays. Bioinformatics 2014; 30: 1363-1369.

20 Pidsley R, Y Wong CC, Volta M, et al. A data-driven approach to preprocessing Illumina 450K methylation array data. BMC Genomics 2013; 14: 293.

21 Anders S, Pyl PT, Huber W. HTSeq - a Python framework to work with high-throughput sequencing data. Bioinformatics 2015; 31: 166-169.

22 Smyth GK. Linear models and empirical Bayes methods for assessing differential expression in microarray experiments. Stat Appl Genet Mol Biol 2004; 3: Article 3.

23 Ritchie ME, Phipson B, Wu D, et al. Limma powers differential expression analyses for RNA-sequencing and microarray studies. Nucleic Acids Res 2015; 43: e47. 

levels by microarray analysis. BMC Bioinformatics 2010; 11: 587. Lehne B, Drong AW, Loh M, et al. A coherent approach for analysis of the Illumina HumanMethylation450 BeadChip improves data quality and performance in epigenome-wide association studies. Genome Biol 2015 ; 16 : 37.

26 Benjamini Y, Hochberg Y. Controlling the false discovery rate: a practical and powerful approach to multiple testing. J R Statistic Soc 1995; 57: 289-300.

27 Kent WJ, Sugnet CW, Furey TS, et al. The human genome browser at UCSC. Genome Res 2002; 12: 996-1006.

28 Pedersen BS, Schwartz DA, Yang IV, et al. Comb-p: software for combining, analyzing, grouping and correcting spatially correlated P-values. Bioinformatics 2012; 28: 2986-2988.

29 Shabalin AA. Matrix eQT: ultra fast eQTL analysis via large matrix operations. Bioinformatics 2012; 28: $1353-1358$.

30 McCarthy DJ, Chen Y, Smyth GK. Differential expression analysis of multifactor RNA-Seq experiments with respect to biological variation. Nucleic Acids Res 2012; 40: 4288-4297.

31 Teschendorff AE, Zheng SC. Cell-type deconvolution in epigenome-wide association studies: a review and recommendations. Epigenomics 2017; 9: 757-768.

32 Michaud J, Simpson KM, Escher R, et al. Integrative analysis of RUNX1 downstream pathways and target genes. BMC Genomics 2008; 9: 363.

33 Vieira Braga FA, Kar G, Berg M, et al. A cellular census of human lungs identifies novel cell states in health and in asthma. Nat Med 2019; 25: 1153-1163.

34 Mukhopadhyay R, Jia J, Arif A, et al. The GAIT system: a gatekeeper of inflammatory gene expression. Trends Biochem Sci 2009; 34: 324-331.

35 Jamieson T, Cook DN, Nibbs RJB, et al. The chemokine receptor D6 limits the inflammatory response in vivo. Nat Immunol 2005; 6: 403-411.

36 Whitehead GS, Wang T, DeGraff LM, et al. The chemokine receptor D6 has opposing effects on allergic inflammation and airway reactivity. Am J Respir Crit Care Med 2007; 175: 243-249.

37 Kontakioti E, Domvri K, Papakosta D, et al. HLA and asthma phenotypes/endotypes: a review. Hum Immunol 2014; 75: 930-939.

38 Topham MK, Prescott SM. Mammalian diacylglycerol kinases, a family of lipid kinases with signaling functions. J Biol Chem 1999; 274: 11447-11450.

39 Yamamoto M, Tanaka T, Hozumi Y, et al. Expression of mRNAs for the diacylglycerol kinase family in immune cells during an inflammatory reaction. Biomed Res 2014; 35: 61-68.

40 Joehanes R, Just AC, Marioni RE, et al. Epigenetic signatures of cigarette smoking. Circ Cardiovasc Genet 2016; 9 : 436-447.

41 Manly BFJ. Randomization, Bootstrap and Monte Carlo Methods in Biology. London, Chapman and Hall, 1997. 